\title{
Development of Sand Volume Estimator for Under-Struck Excavator Bucket Using Single Camera
}

In-Hwan Kim, Dong-Woo Lim, and Jin-Woo Jung

Department of Computer Science and Engineering, Dongguk University, Seoul, Korea

\section{]jfis}

\begin{abstract}
To support the intelligence of construction environment, it is important to measure the workload of excavator in real time. But, previous studies are expensive to implement or can not processed in real time. In this paper, an image-based method to estimate the workload of the excavator bucket especially for the state of under-struck is proposed by assuming the shape of bucket and the shape of sand inside the bucket as geometric models. By analyzing the relation between single camera image and actual bucket geometry, the volume of sand which is proportional to the excavator workload is estimated. The experimental results show $93.5 \%$ accuracy even though only some part of sand region is unseen.
\end{abstract}

Keywords: Sand volume estimator, Excavator bucket modeling, Single camera

\section{Introduction}

It is not easy to measure in real time the amount of sand which an excavator load in working environment [1, 2]. Previous method to measure this workload in real environment was to measure the charge of weight of dump truck. In order to make this weight change, it is needed to stop the truck in a certain area. However, this method is inefficient in terms of time because it decreases the working efficiency by stopping the truck. This paper proposes a new method which can estimate automatically the volume of sand in a bucket by capturing an image from a single camera in the excavator and analyzing the image specifically focused on the under-struck state.

\section{Background}

Depending on the amount of sand piled up in a bucket of excavator, states could be divided like Figure 1. It is called as struck state if the volume of bucket and that of sand are equal. It is called as under-struck sate if the volume of sand is less than struck state. If the volume of sand is larger than struck state, it is called as heaped state. This paper focuses on under-struck state. The purpose of this paper is to estimate the amount of sand from the bucket image based on a single camera image processing [3, 4].

This paper assumes A1-A4 to estimate the volume of under-struck state.

A1: The shape of bucket is modeled as a combination of half cylinder and right triangular prism. 


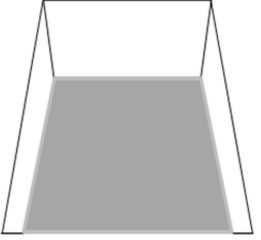

(a)

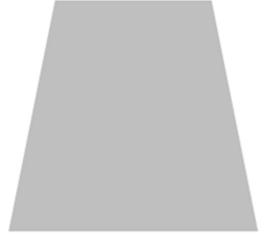

(b)

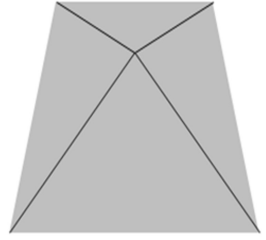

(c)
Figure 1. Three representative states of sands in the bucket. (a) under-struck state, (b) struck state, and (c) heaped state.

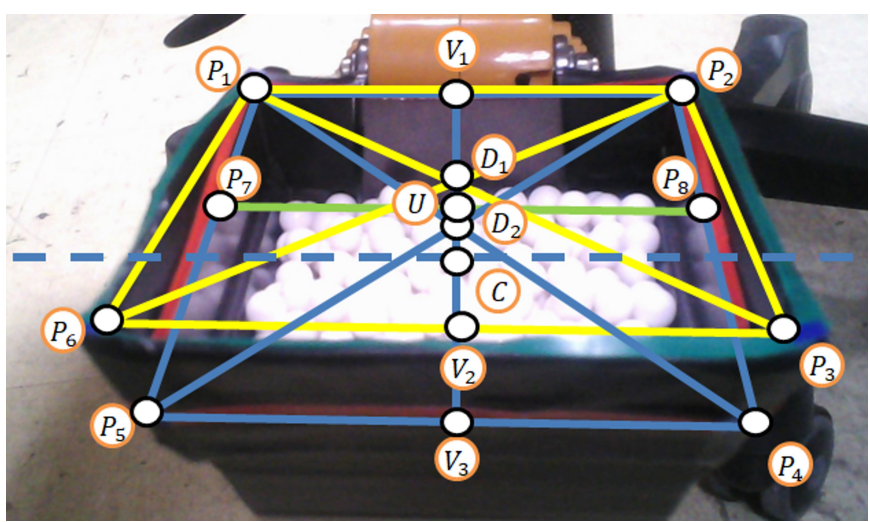

Figure 2. Bucket image.

A2: The bucket diameter '2a', bucket width ' $b$ ' and bucket teeth length 'c' are given by the excavator company.

A3: When the image is captured, the upper side of bucket is horizontal to the ground.

A4: In Figure 2 , the pixel locations of points $\left(P_{1}\right.$ to $\left.P_{8}\right)$ and the highest vertical line (U) can be calculated with image processing [5, 6].

\section{Single Camera-based Sand Volume Estima- tion Algorithm for Under-Struck State}

Under-struck state means that the highest line of sand $U$ is below the line (Figure 3). This state could be divided into three cases in order to reduce the complexity of the formula. (Figure 2) The condition dividing the under-struck state is as follows:

L1: line segment $\overline{A B}$

L2: parallel line segment with $\overline{A B}$ passing through $\mathrm{O}$

L3: Parallel line segment with $\overline{A B}$ passing through D

If $(U<L 3)$ state $\leftarrow$ Under-Struck-Case1

Else if $(U<L 2)$ state $\leftarrow$ Under-Struck-Case2

Else if $(U<L 1)$ state $\leftarrow$ Under-Struck-Case3

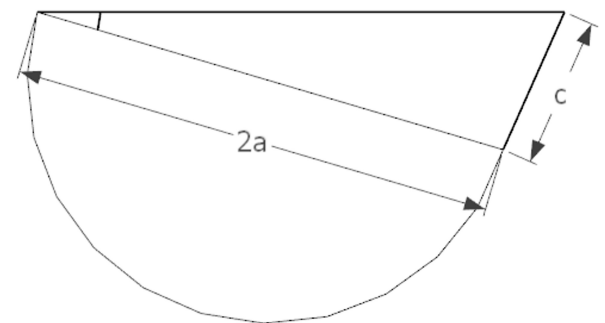

Figure 3. Bucket modeling.

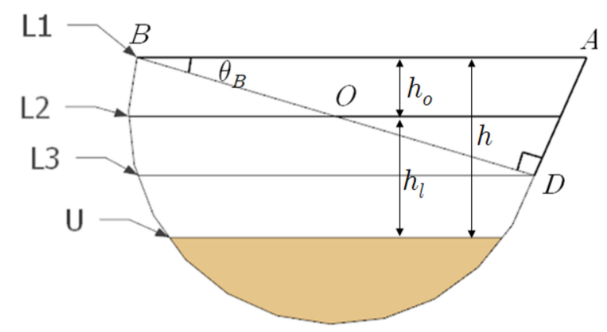

Figure 4. Three cases for under-struck state.

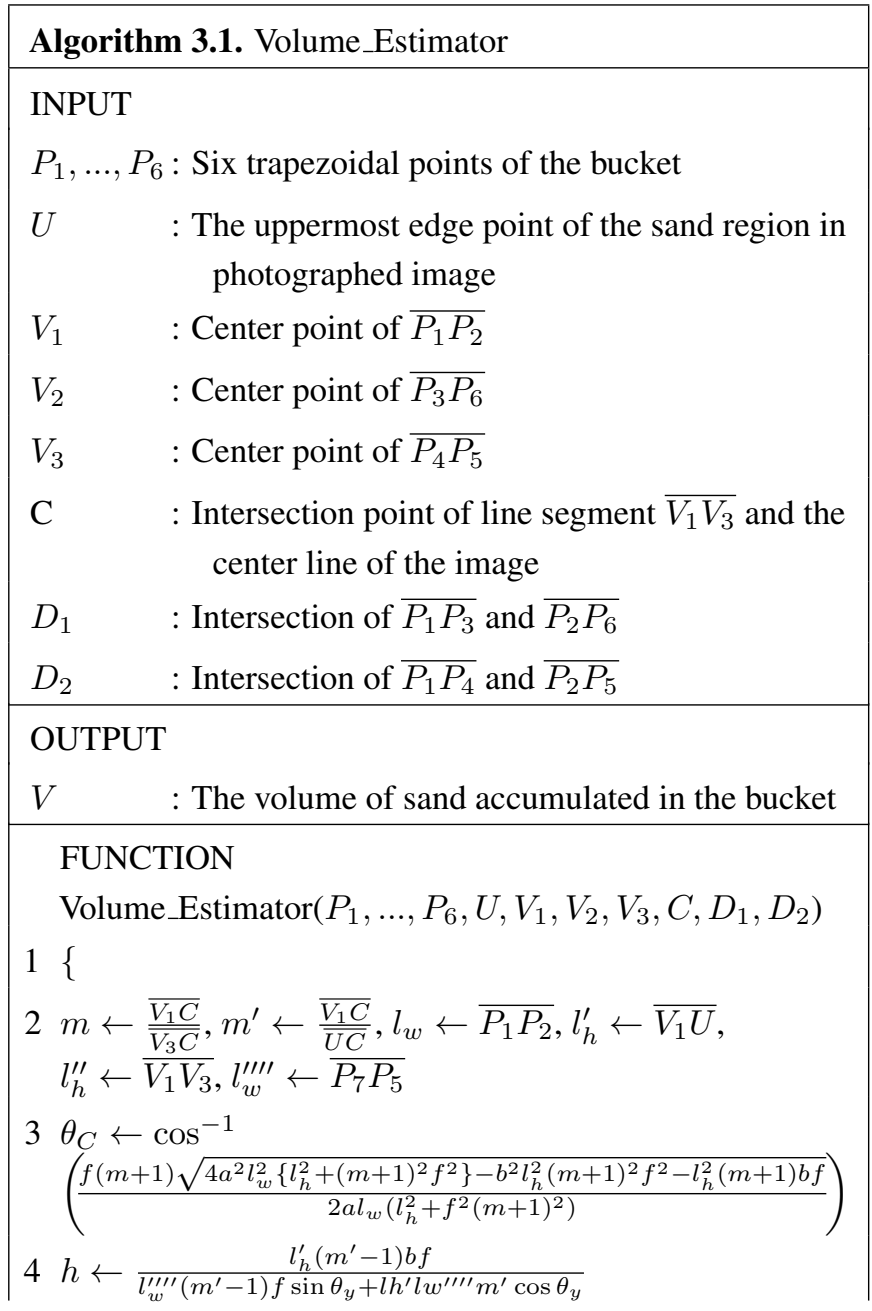


$5 h \leftarrow \frac{l_{h}^{\prime}\left(m^{\prime}-1\right) b f}{l_{w}^{\prime \prime \prime \prime}\left(m^{\prime}-1\right) f \sin \theta_{y}+l h^{\prime} l w^{\prime \prime \prime \prime} m^{\prime} \cos \theta_{y}}$

6 IF $\left(h \geq h_{0}+a \sin \theta_{B}\right)$ THEN

$7 \quad$ state $\leftarrow$ Under-Struck-Case 1

8 ELSE IF $\left(h_{0}+a \sin \theta_{B}>h \geq h_{0}\right)$ THEN

9 stae $\leftarrow$ Under-Struck-Case2

10 ELSE IF $\left(h<h_{0}\right)$ THEN $\quad / / h_{0}=a \sin \theta_{B}$

11 state $\leftarrow$ Under-Struck-Case3

12 END IF

13 IF (state $=$ Under-Struck-Case 1$)$ THEN

$14 V \leftarrow a^{2} b \cos ^{-1}\left(\frac{h_{l}}{a}\right)-b h_{l} \sqrt{a^{2}-h_{l}^{2}}$

15 ELSE IF (state=Under-Struck-Case2) THEN

$16 V \leftarrow \frac{\pi a^{2} b}{2}-\frac{a^{2} b}{2}\left(\theta_{B}+\frac{\pi}{2}-\cos ^{-1}\left(\frac{h_{l}}{a}\right)\right)$

$$
\begin{aligned}
& -\frac{b h_{l}}{2}\left(\sqrt{a^{2}-h_{l}^{2}}+h_{l} \frac{\cos \theta_{B}}{\sin \theta_{B}}\right) \\
& +\frac{b}{2} \tan \theta_{B}\left(\frac{a \sin \theta_{B}-h_{l}}{\sin \theta_{B}}\right)^{2}
\end{aligned}
$$

17 ELSE IF (state=Under-Struck-Case3) THEN

$$
\begin{aligned}
18 \leftarrow & \frac{\pi a^{2} b}{2}-\frac{b}{2} \tan \theta_{B}\left(\frac{2 a \sin \theta_{B}-h}{\sin \theta_{B}}\right)^{2} \\
& -\frac{a^{2} b}{2}\left(\theta_{B}-\cos ^{-1}\left(\frac{\sqrt{a^{2}-\left(a \sin \theta_{B}-h\right)^{2}}}{a}\right)\right) \\
& +\frac{b}{2}\left(a \sin \theta_{B}-h\right)\left(\sqrt{a^{2}-\left(a \sin \theta_{B}-h\right)^{2}}-\frac{a \sin \theta_{B}-h}{\tan \theta_{B}}\right)
\end{aligned}
$$

19 END IF

\section{RETURN V}

$21\}$

\subsection{Determination of Under-Struck State Parameters}

Specific parameters are required to estimate the volume in case of Under-Struck State. There are parameters that need to be known by default such as $l_{w}, l_{w}^{\prime}, l_{w}^{\prime \prime \prime \prime}, l_{h}^{\prime}, l_{h}^{\prime \prime \prime}$, and so on. These basic parameters can be obtained using each point $\left(P_{1} \sim P_{8}, U\right)$ through image processing.

In Figure 5,

$$
\begin{aligned}
& l_{w}=\sqrt{\left(\alpha_{2}-\alpha_{1}\right)^{2}+\left(\beta_{2}-\beta_{1}\right)^{2}}, \\
& l_{w}^{\prime \prime \prime \prime}=\sqrt{\left(\alpha_{8}-\alpha_{7}\right)^{2}+\left(\beta_{8}-\beta_{7}\right)^{2}}, \\
& l_{h}^{\prime \prime \prime \prime}=\sqrt{\left(\alpha_{7}-\alpha_{1}\right)^{2}+\left(\beta_{7}-\beta_{1}\right)^{2}-\left(\frac{l_{w}-l_{w}^{\prime \prime \prime \prime}}{2}\right)^{2}}, \\
& l_{h}^{\prime \prime \prime}=\sqrt{\left(\alpha_{5}-\alpha_{1}\right)^{2}+\left(\beta_{5}-\beta_{1}\right)^{2}-\left(\frac{l_{w}-l_{w}^{\prime \prime \prime}}{2}\right)^{2}} .
\end{aligned}
$$

The unit of parameters $l_{w}, l_{w}^{\prime \prime \prime \prime}, l_{h}^{\prime}, l_{h}^{\prime \prime \prime}$ are number of pixels. In order to calculate the volume, metric unit such as $\mathrm{mm}$ is

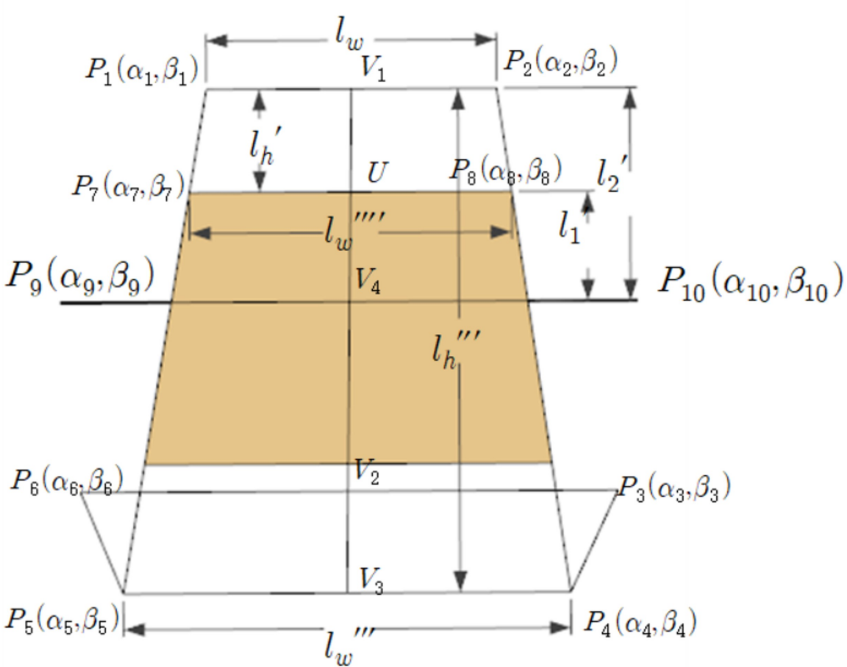

Figure 5. Default parameters for under-struck state.

needed instead of number of pixels. This conversion is possible by multiplying the actual pixel size of image sensor.

\subsection{Calculation of Angle between the Camera Centre Line $\overline{Z F}$ and the Bucket Line $\overline{A B}$}

We can not always take the picture at the same angle. That changes the parameter values of bucket. This paper use the expression of the angle between the camera centre line and the bucket to solve this problem. This is called $\theta_{C}$. This section will describe the process of calculating $\theta_{C}$.

In Figure 6, lets assume that

$$
r_{2}=k r_{1}
$$

By the similarity between $\triangle E D D^{\prime}$ and $\triangle E C^{\prime} F$,

$$
\begin{aligned}
& 2 a \cos \theta_{c}: r_{1}=d:\left(r_{1}+r_{2}\right)=d:(1+k) r_{1}, \\
& k=\frac{d}{2 a \cos \theta_{C}}-1 .
\end{aligned}
$$

In Figure 6,

$L_{1}=2 a \sin \theta_{c}-k r_{1}=2 a \sin \theta_{c}-\left(\frac{d}{2 a \cos \theta_{c}}-1\right) r_{1}$

By the similarity between $\triangle F Z Y$ and $\triangle F C^{\prime} B$,

$$
\begin{aligned}
& d: f=L_{1}: l_{2}, \\
& l_{2}=\frac{f}{d}\left[2 a \sin \theta_{c}-\left(\frac{d}{2 a \cos \theta_{c}}-1\right) r_{1}\right],
\end{aligned}
$$




$$
\begin{aligned}
& d: f=(1+k) r_{1}: l_{1}=\frac{d r_{1}}{2 a \cos \theta_{c}}: l_{1}, \\
& l_{1}=\frac{f r_{1}}{2 a \cos \theta_{c}} .
\end{aligned}
$$

$l_{h}^{\prime \prime \prime}$ is the height of bucket in Figure 5.

$$
\begin{aligned}
& l_{h}^{\prime \prime \prime}=l_{1}+l_{2}=\frac{f}{d}\left(2 a \sin \theta_{c}+r_{1}\right), \\
& r_{1}=\frac{d l_{h}^{\prime \prime \prime}}{f}-2 a \sin \theta_{c}, \\
& l_{1}=\frac{d l_{h}^{\prime \prime \prime}}{2 a \cos \theta_{c}}-f \tan \theta_{c}, \\
& l_{2}=l_{h}^{\prime \prime \prime}+f \tan \theta_{c}-\frac{d l_{h}}{2 a \cos \theta_{c}} .
\end{aligned}
$$

$l_{1}$ and $l_{2}$ are decided based on the centerline of the camera image. Lets assume that the ratio of $l_{2}$ and $l_{1}$ is $m$.

$$
\begin{aligned}
& l_{2}=m l_{1}, \\
& l_{h}^{\prime \prime \prime}=(1+m) l_{1}, \\
& l_{1}=\frac{l_{h}^{\prime \prime \prime}}{1+m} .
\end{aligned}
$$

By Eq. 15] and Eq. [19],

$$
l_{h}^{\prime \prime \prime}=\frac{2 a f(m+1) \sin \theta_{c}}{(m+1) d-2 a \cos \theta_{c}} .
$$

By Figure 5 and Figure 6,

$$
\begin{aligned}
& b: d=l_{w}: f, \\
& d=\frac{b f}{l_{w}} .
\end{aligned}
$$

By Eq. 20, and Eq. 22,

$$
\begin{aligned}
& l_{h}^{\prime \prime \prime}=\frac{2 a l_{w} f(m+1) \sin \theta_{c}}{(m+1) b f-2 a l_{w} \cos \theta_{c}} \\
& \theta_{c} \\
& =\cos ^{-1}\left(\frac{\sqrt{4 a^{2} l_{w}^{2}\left(l^{\prime \prime \prime}{ }_{h}^{2}+(m+1)^{2} f^{2}\right)-b^{2} l^{\prime \prime \prime}{ }_{h}^{2}(m+1)^{2} f^{2}}}{2 a l_{w}\left(l^{\prime \prime \prime}{ }_{h}^{2}+f^{2}(m+1)^{2}\right)}\right. \\
& \left.\quad \times f(m+1)-\frac{{l^{\prime \prime \prime}}_{h}^{2}(m+1) b f}{2 a l_{w}\left(l^{\prime \prime \prime}{ }_{h}^{2}+f^{2}(m+1)^{2}\right)}\right)
\end{aligned}
$$

\subsection{Calculation of Angle $\theta_{B}$ between $\overline{A B}$ and $\overline{B D}$}

In Figure 3, $\theta_{B}$ is fixed angle so it can be obtained by Eq. 25.

$$
\theta_{B}=\tan ^{-1}\left(\frac{c}{2 a}\right)
$$

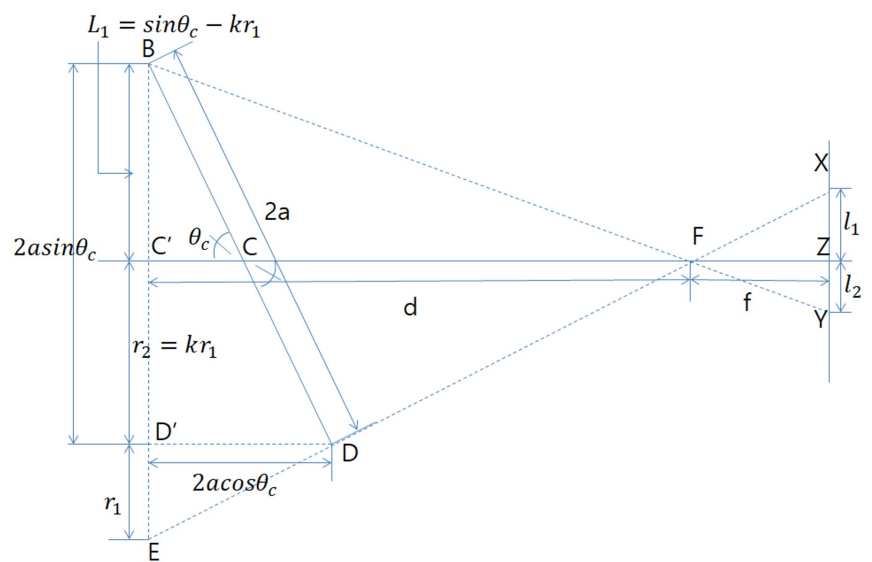

Figure 6. Camera geometry for calculating $\theta_{C}$.

\subsection{Calculation of Height $h$ between the Bucket line $\overline{A B}$ and $\overline{D H}$}

$\theta_{y}$ can be obtained by using $\theta_{C}$ and $\theta_{B}$.

$$
\theta_{y}=\frac{\pi}{2}-\theta_{C}+\theta_{B}
$$

In Figure 7, lets assume that

$$
r_{2}^{\prime}=k^{\prime} r_{1}^{\prime}
$$

The value $k^{\prime}$ can be obtained by using the similarity of $\triangle E B^{\prime} H^{\prime}$ and $\triangle E C^{\prime} F$.

$$
\begin{aligned}
& h \cos \theta_{y}: r_{1}^{\prime}=d^{\prime}:\left(r_{1}^{\prime}+r_{2}^{\prime}\right)=d^{\prime}:\left(1+k^{\prime}\right) r_{1}^{\prime}, \\
& k^{\prime}=\frac{d^{\prime}}{h \cos \theta_{y}}-1 .
\end{aligned}
$$

By the similarity between $\triangle F E C^{\prime}$ and $\triangle F Y Z$

$$
\begin{aligned}
& d^{\prime}: f=\frac{d^{\prime} r_{1}^{\prime}}{h \cos \theta_{y}}: l_{2}^{\prime}, \\
& l_{2}^{\prime}=\frac{f r_{1}^{\prime}}{h \cos \theta_{y}} .
\end{aligned}
$$

By the similarity between $\triangle F H C^{\prime}$ and $\triangle F X A$.

$d^{\prime}: f=L_{1}^{\prime}: l_{1}^{\prime}$,

$L_{1}^{\prime}=k^{\prime} r_{1}^{\prime}-h \sin \theta_{y}=\left(\frac{d^{\prime}}{h \cos \theta_{y}}-1\right) r_{1}^{\prime}-h \sin \theta_{y}$,

$l_{1}^{\prime}=\frac{f}{d^{\prime}}\left[\left(\frac{d^{\prime}}{h \cos \theta_{y}}-1\right) r_{1}^{\prime}-h \sin \theta_{y}\right]$. 


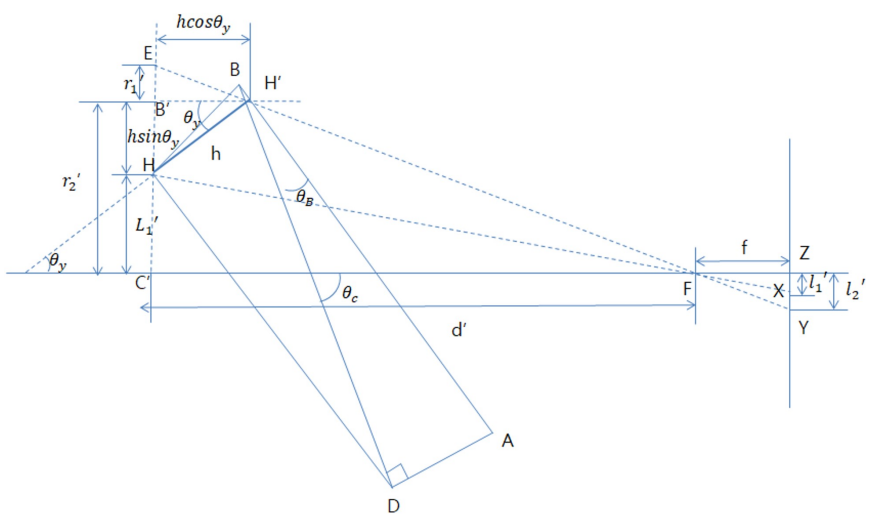

Figure 7. Camera geometry for calculating $h$.

In Figure 5,

$$
\begin{aligned}
& l_{h}^{\prime}=l_{2}^{\prime}-l_{1}^{\prime}=\frac{f}{d^{\prime}}\left(h \sin \theta_{y}+r_{1}^{\prime}\right), \\
& r_{1}^{\prime}=\frac{d^{\prime} l_{h}^{\prime}}{f}-h \sin \theta_{y} .
\end{aligned}
$$

By Eq. (34) and Eq. (36),

$$
l_{1}^{\prime}=\frac{d^{\prime} l_{h}^{\prime}}{h \cos \theta_{y}}-l_{h}^{\prime}-f \tan \theta_{y} .
$$

Let's assume that

$$
\begin{aligned}
& l_{2}^{\prime}=m^{\prime} l_{1}^{\prime}, \\
& l_{h}^{\prime}=\left(m^{\prime}-1\right) l_{1}^{\prime}, \\
& l_{1}^{\prime}=\frac{l_{h}^{\prime}}{m^{\prime}-1} .
\end{aligned}
$$

In Figure 5,

$$
\begin{aligned}
& b: d^{\prime}=l_{w}^{\prime \prime \prime \prime}: f \\
& d^{\prime}=\frac{b f}{l_{w}^{\prime \prime \prime \prime}} .
\end{aligned}
$$

By Eq. (37), Eq. (40), and Eq. (42),

$$
\begin{aligned}
& l_{h}^{\prime}=\frac{l_{w}^{\prime \prime \prime \prime}\left(m^{\prime}-1\right) f h \sin \theta_{y}}{\left(m^{\prime}-1\right) b f-l_{w}^{\prime \prime \prime \prime} m^{\prime} h \cos \theta_{y}} \\
& h=\frac{l_{h}^{\prime}\left(m^{\prime}-1\right) b f}{l_{w}^{\prime \prime \prime \prime}\left(m^{\prime}-1\right) f \sin \theta_{y}+l_{h}^{\prime} l_{w}^{\prime \prime \prime \prime} m^{\prime} \cos \theta_{y}} \\
& h_{l}=h-h_{o} \\
& h_{o}=a \sin \theta_{B}, \\
& h_{l}=\frac{l_{h}^{\prime}\left(m^{\prime}-1\right) b f}{l_{w}^{\prime \prime \prime \prime}\left(m^{\prime}-1\right) f \sin \theta_{y}+l_{h}^{\prime} l_{w}^{\prime \prime \prime \prime} m^{\prime} \cos \theta_{y}}-a \sin \theta_{B}
\end{aligned}
$$

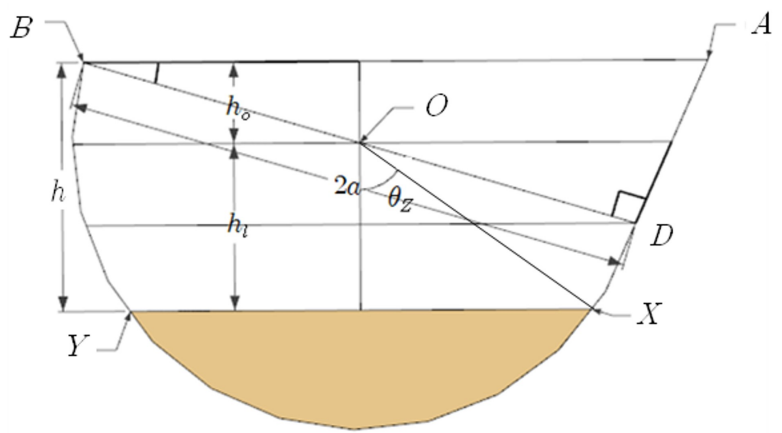

Figure 8. Under-struck-case1.

\subsection{Estimation of Under-Struck State Volume}

\subsubsection{Estimation of sand volume for Under-struck-case 1 condition}

The volume can be estimated by sector $O X Y$ and $\triangle O Y X$. Here, $V(x)$ means volume made by expanding the area $x$ along bucket width $b$.

$$
\begin{aligned}
& V=V(\text { sector } O X Y)-V(\triangle O Y X), \\
& \theta_{z}=\cos ^{-1}\left(\frac{h_{l}}{a}\right) .
\end{aligned}
$$

By Eq. 49,

$$
\begin{aligned}
& \text { sector } O X Y=a^{2} \cos ^{-1}\left(\frac{h_{l}}{a}\right), \\
& \overline{X Y}=2 \sqrt{a^{2}-h_{l}^{2}}, \\
& \triangle O Y X=h_{l} \sqrt{a^{2}-h_{l}^{2}}, \\
& V=a^{2} b \cos ^{-1}\left(\frac{h_{l}}{a}\right)-b h_{l} \sqrt{a^{2}-h_{l}^{2}} .
\end{aligned}
$$

\subsubsection{Estimation of sand volume for under-struck-case 2 condition}

In under-struck-case 2 , the formula for calculating the volume can be obtained by subtracting sector $B O Y$ and $\triangle Y O C$ from sector $B D O$ and adding $\triangle X D C$. See Figure 9.

$$
\begin{aligned}
V= & V(\text { semicircle })-V(\text { sector } B O Y)-V(\triangle Y O C) \\
& +V(\triangle X D C) .
\end{aligned}
$$

First, to obtain sector $B O Y$, we need to find $u 1$ and $u 2$.

$$
u 1=\theta_{B}
$$




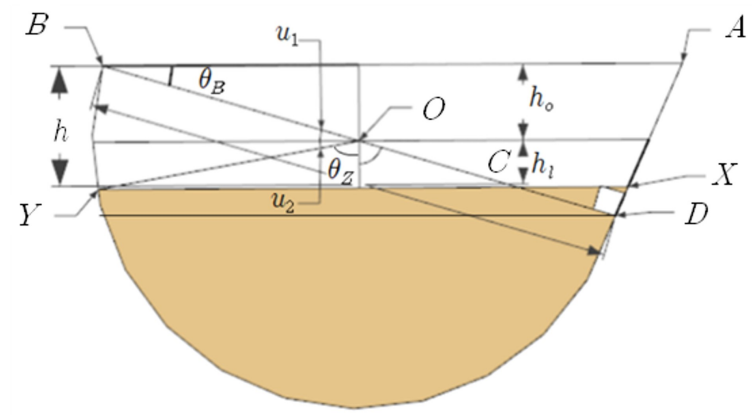

Figure 9. Under-struck-case2.

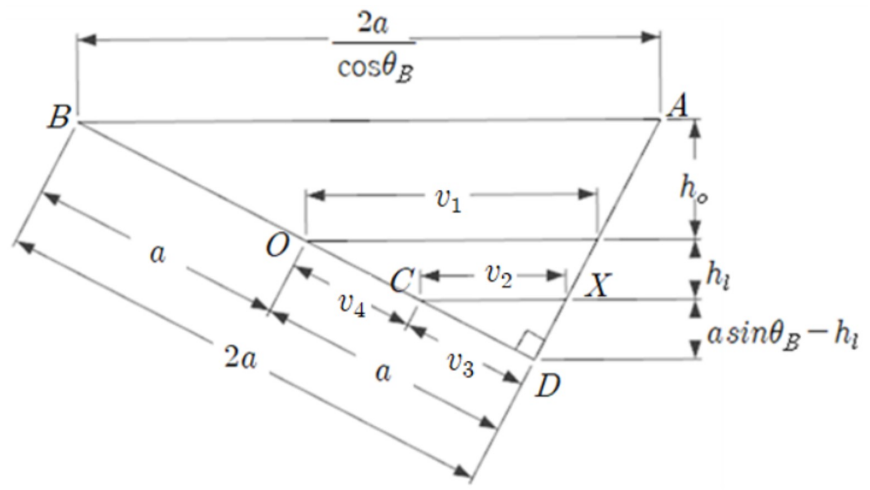

Figure 10. $\triangle X D C$.

$$
\begin{aligned}
& u 2=\frac{\pi}{2}-\cos ^{-1}\left(\frac{h_{l}}{a}\right), \\
& \text { sector } B O Y=\frac{a^{2}}{2}\left(\theta_{B}+\frac{\pi}{2}-\cos ^{-1}\left(\frac{h_{l}}{a}\right)\right) .
\end{aligned}
$$

To find the area of $\triangle X D C$, you need to find the base length and height. In Figure 10,

$$
\begin{aligned}
& \frac{2 a}{\cos \theta_{B}}: h_{o}+a \sin \theta_{B}=v_{2}: a \sin \theta_{B}-h_{l}, \\
& 2 a: h_{o}+a \sin \theta_{B}=v_{3}: a \sin \theta_{B}-h_{l} .
\end{aligned}
$$

By Eq. [46,

$$
\begin{aligned}
& v_{2}=\frac{a \sin \theta_{B}-h_{l}}{\sin \theta_{B} \cos \theta_{B}}, \\
& v_{3}=a-\frac{h_{l}}{\sin \theta_{B}}, \\
& \triangle X D C=\frac{1}{2} v_{3} \sqrt{v_{2}^{2}-v_{3}^{2}}=\frac{1}{2} \tan \theta_{B}\left(\frac{a \sin \theta_{B}-h_{l}}{\sin \theta_{B}}\right)^{2} .
\end{aligned}
$$

In Figure 10,

$$
v_{4}=a-v_{3} .
$$

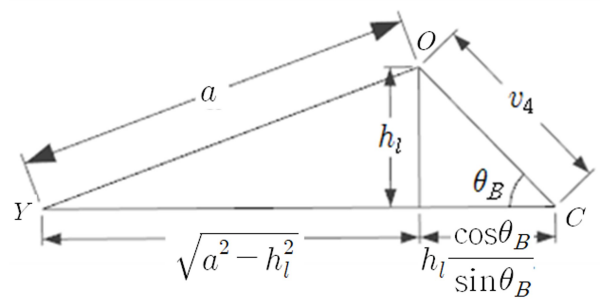

Figure 11. $\triangle Y O C$.

In Figure 9 and Figure 11,

$$
\triangle Y O C=\frac{1}{2} h_{l}\left(\sqrt{a^{2}-h_{l}^{2}}+h_{l} \frac{\cos \theta_{B}}{\sin \theta_{B}}\right),
$$

The total volume can be obtained by summing each areas.

$$
\begin{aligned}
V= & \frac{\pi a^{2} b}{2}-\frac{a^{2} b}{2}\left(\theta_{B}+\frac{\pi}{2}-\cos ^{-1}\left(\frac{h_{l}}{a}\right)\right) \\
& -\frac{b h_{l}}{2}\left(\sqrt{a^{2}-h_{l}^{2}}+h_{l} \frac{\cos \theta_{B}}{\sin \theta_{B}}\right) \\
& +\frac{b}{2} \tan \theta_{B}\left(\frac{a \sin \theta_{B}-h_{l}}{\sin \theta_{B}}\right)^{2} .
\end{aligned}
$$

\subsubsection{Estimation of sand volume for under-struck-case 3 condition}

The equation for obtaining the volume in under-struck-case 3 can be obtained by adding $\triangle Y^{\prime} Y O$ and $\triangle X Y^{\prime} D$ in the semicircle-subtracted sector $B Y O$. See Figure 12.

$$
\begin{aligned}
V= & V(\text { semicircle })-V(\text { sector } B Y O) \\
& +V\left(\triangle Y^{\prime} Y O\right)+V\left(\triangle X Y^{\prime} D\right) .
\end{aligned}
$$

In Figure 13,

$$
\begin{aligned}
& 2 a: 2 a \sin \theta_{B}=2 a-v_{5}: 2 a \sin \theta_{B}-h, \\
& \frac{2 a}{\cos \theta_{B}}: 2 a \sin \theta_{B}=v_{6}: 2 a \sin \theta_{B}-h, \\
& v_{5}=\frac{h}{\sin \theta_{B}}, \\
& v_{6}=\frac{2 a \sin \theta_{B}-h}{\sin \theta_{B} \cos \theta_{B}}, \\
& \triangle X Y^{\prime} D=\frac{1}{2} \tan \theta_{B}\left(\frac{2 a \sin \theta_{B}-h}{\sin \theta_{B}}\right)^{2} .
\end{aligned}
$$

In Figure 14,

$$
\begin{aligned}
& v_{8}=\sqrt{\left(a-v_{5}\right)^{2}-\left(a \sin \theta_{B}-h\right)^{2}}, \\
& v_{7}=\sqrt{a^{2}-\left(a \sin \theta_{B}-h\right)^{2}}-v_{8},
\end{aligned}
$$




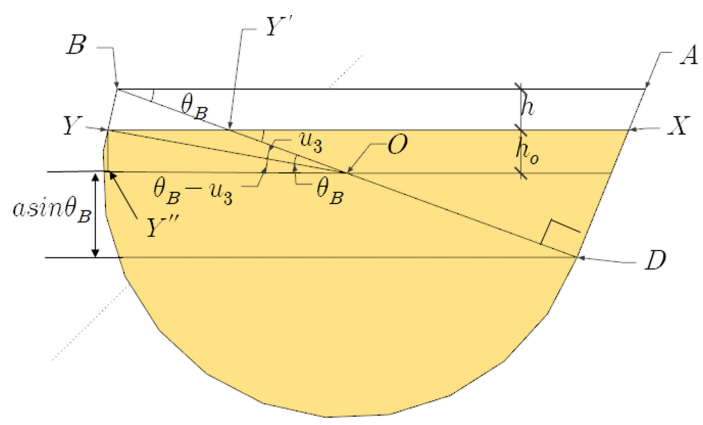

Figure 12. Under-struck-case3.

$$
v_{7}+v_{8}=\sqrt{a^{2}-\left(a \sin \theta_{B}-h\right)^{2}} .
$$

In Figure 15,

$\theta_{B}-u_{3}=\cos ^{-1}\left(\frac{\sqrt{a^{2}-\left(a \sin \theta_{B}-h\right)^{2}}}{a}\right)$,
$u_{3}=\theta_{B}-\cos ^{-1}\left(\frac{\sqrt{a^{2}-\left(a \sin \theta_{B}-h\right)^{2}}}{a}\right)$,

sector $B Y O=\frac{a^{2}}{2}\left(\theta_{B}-\cos ^{-1}\left(\frac{\sqrt{a^{2}-\left(a \sin \theta_{B}-h\right)^{2}}}{a}\right)\right)$.

In Figure 16,

$\triangle Y^{\prime} Y O$

$=\frac{1}{2}\left(a \sin \theta_{B}-h\right)\left(\sqrt{a^{2}-\left(a \sin \theta_{B}-h\right)^{2}}-\frac{a \sin \theta_{B}-h}{\tan \theta_{B}}\right)$,

V

$$
\begin{aligned}
= & \frac{\pi a^{2} b}{2}+\frac{b}{2} \tan \theta_{B}\left(\frac{2 \operatorname{asin} \theta_{B}-h}{\sin \theta_{B}}\right)^{2} \\
& -\frac{a^{2} b}{2}\left(\theta_{B}-\cos ^{-1}\left(\frac{\sqrt{a^{2}-\left(a \sin \theta_{B}-h\right)^{2}}}{a}\right)\right) \\
& +\frac{b}{2}\left(a \sin \theta_{B}-h\right)\left(\sqrt{a^{2}-\left(a \sin \theta_{B}-h\right)^{2}}-\frac{a \sin \theta_{B}-h}{\tan \theta_{B}}\right) .
\end{aligned}
$$

\section{Experimental Results}

Several experiments were performed to confirm the accuracy of the algorithm presented in this paper. The volume expression contains a parameter called focal length. This focal length parameter may or may not appear in the $\mathrm{H} / \mathrm{W}$ specification of commercial camera. Here, we assumed that the focal length is

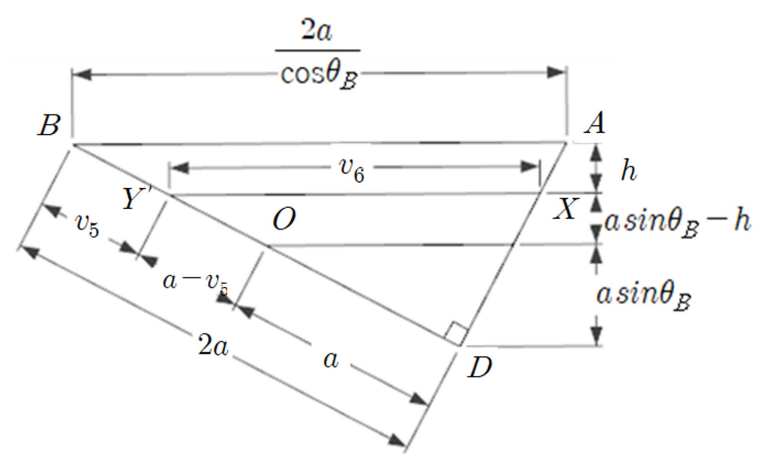

Figure 13. $\triangle X Y^{\prime} D$.

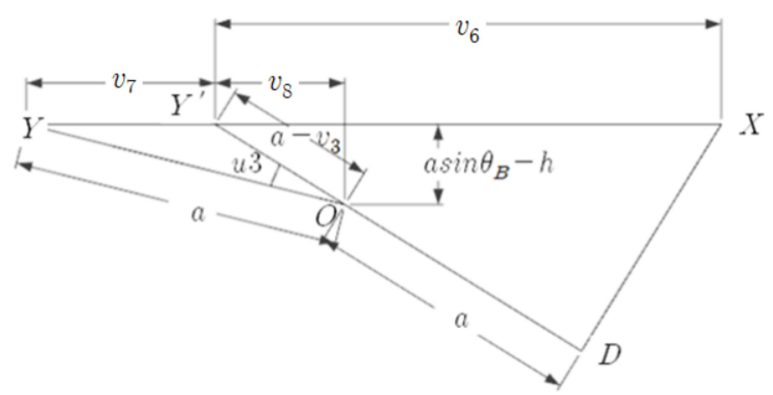

Figure 14. Sector $B Y O-1$.

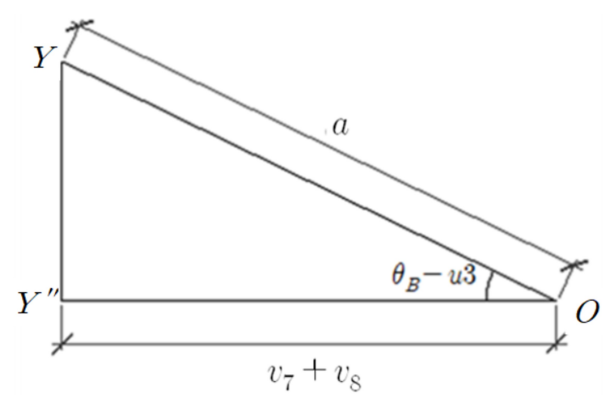

Figure 15. Sector $B Y O-2$.

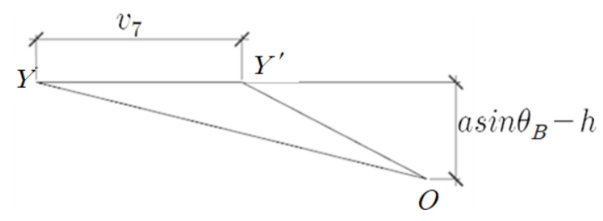

Figure 16. $\triangle Y^{\prime} Y O$.

not given and try to find by experiments using pre-known $l_{w}$. The estimated focal length was $19.0011 \mathrm{~mm}$ as Table 1 when we use SPC-B900W camera. The size of image sensor in the camera was $21.12 \mathrm{~mm} \times 11.88 \mathrm{~mm}$.

The experiment to find the focal length was done by taking a picture of an object with pre-known size at a pre-known distance. Then the focal length is found through the number of 
Table 1. Focal length experiment

\begin{tabular}{cccc}
\hline $\begin{array}{c}\text { Length } \\
\text { between object } \\
\text { and camera } \\
\text { lens } \\
(\mathrm{mm})\end{array}$ & $\begin{array}{c}\text { Object length } \\
(\mathrm{mm})\end{array}$ & $\begin{array}{c}\text { Image pixel } \\
\text { length } \\
\text { (pixel) }\end{array}$ & $\begin{array}{c}\text { Focal length } \\
(\mathrm{mm})\end{array}$ \\
\hline 450 & 113 & 441 & 19.3181 \\
400 & 113 & 499 & 19.4301 \\
350 & 113 & 559 & 19.0456 \\
300 & 113 & 652 & 19.0407 \\
\hline 250 & 113 & 783 & 19.0553 \\
\hline 200 & 113 & 963 & 18.7487 \\
150 & 113 & 1258 & 18.3690 \\
\hline & Average focal length & 19.0011 \\
\hline
\end{tabular}

Table 2. Density experiment of object

\begin{tabular}{cccc}
\hline $\begin{array}{c}\text { Number of } \\
\text { balls }\end{array}$ & Volume $(\mathrm{mL})$ & Weight $(\mathrm{g})$ & Density $(\mathrm{g} / \mathrm{mL})$ \\
\hline 10 & 1.25 & 2.0 & 1.6 \\
20 & 2.5 & 4.0 & 1.6 \\
30 & 3.75 & 6.0 & 1.6 \\
40 & 5 & 8.0 & 1.6 \\
\hline & Average density & & 1.6 \\
\hline
\end{tabular}

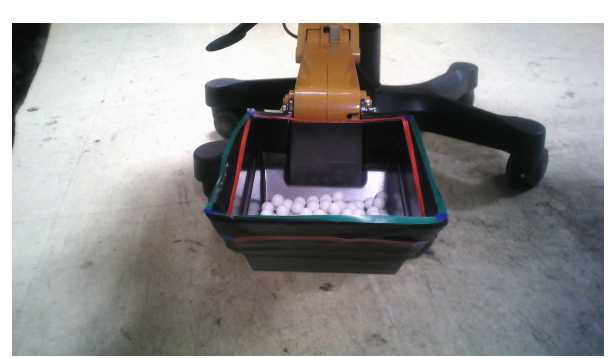

Figure 17. Under-struck-case1 image.

image pixels and pre-known size and distance of the object.

The experiment to find the density of a ball was done by finding the volume and weight of a fixed number of $6 \mathrm{~mm}$ balls. The density is determined by dividing the weight by the measured volume.

The sand used in the experiment is a type of ball with $6 \mathrm{~mm}$ diameter. The method to estimate the volume is as follows: The original weight is obtained through an electronic scale. Actual Volume is the volume measured when pouring water into the beaker and then pouring balls there. Estimated volume is obtained through the proposed algorithm. Estimated weight is obtained by multiplying the estimated volume by the average $1.6 \mathrm{~g} / \mathrm{ml}$ density obtained in Table 2 .

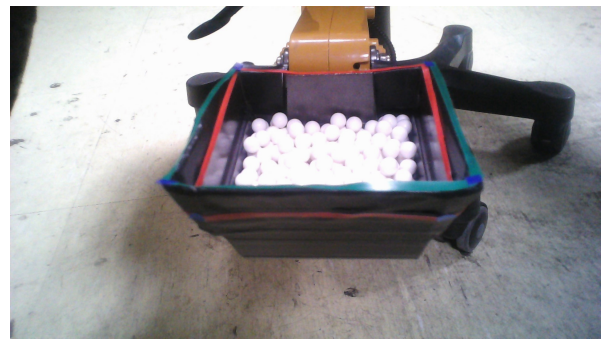

Figure 18. Under-struck-case2 image.

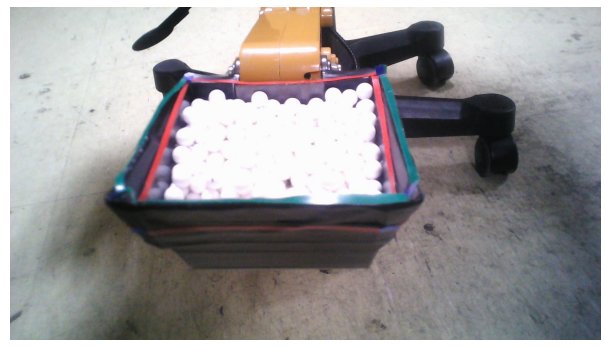

Figure 19. Under-struck-case3 image.

Table 3. Experimental information

\begin{tabular}{ccccccc}
\hline state & $\begin{array}{c}\text { Grain } \\
\text { size } \\
(\mathrm{mm})\end{array}$ & $\begin{array}{c}\text { Original } \\
\text { weight } \\
(\mathrm{g})\end{array}$ & $\begin{array}{c}\text { Actual } \\
\text { volume } \\
(\mathrm{mL})\end{array}$ & $\begin{array}{c}\text { Estimated Estimated } \\
\text { volume } \\
(\mathrm{mL})\end{array}$ & $\begin{array}{c}\text { Error } \\
\text { weight } \\
(\mathrm{g})\end{array}$ & $\begin{array}{c}\text { ratio } \\
(\%)\end{array}$ \\
\hline \multirow{2}{*}{ case1 } & 6 & 39.3 & 24.5 & 22.0 & 35.1 & 10.6 \\
& 6 & 39.3 & 24.5 & 23.1 & 36.9 & 6.1 \\
case2 & 6 & 104.9 & 60 & 59.1 & 94.6 & 9.8 \\
& 6 & 104.9 & 60 & 65.2 & 104.3 & 0.5 \\
case3 & 6 & 153.6 & 95 & 94.1 & 150.5 & 2.0 \\
& 6 & 153.6 & 95 & 105.9 & 169.4 & 10.3 \\
\hline
\end{tabular}

The experimental results are shown in Table 3. The error rate of case 1 was $10.6 \%$ and $6.1 \%$, the error rate of case 2 was $9.8 \%$ and $0.5 \%$, and the error rate of case 3 was $2.0 \%$ and $10.3 \%$. Most of errors may be from the uneven surface of the sand region made by the fixed size of ball.

\section{Conclusions}

In this paper, we proposed a novel method to estimate the amount of sands in the excavator bucket based on a single camera by using the image processing technique and mathematical modeling of bucket. For each of three under-struck states, a closed form of mathematical solution to estimate the sand volume of excavator bucket was implemented. The experimental results show that the error rate is within $10.6 \%$ and the minimum error rate is $0.5 \%$ in a case of under-struck-case 2 . 


\section{Conflict of Interest}

No potential conflict of interest relevant to this article was reported.

\section{Acknowledgements}

This research was partially supported by the MIST (Ministry of Science and ICT), Korea, under the national program for excellence in SW supervised by the IITP (Institute for Information \& Communications Technology Promotion) (2016-0-00017) and partially supported by Basic Science Research Program through the National Research Foundation of Korea (NRF) funded by the Ministry of Education, Science and Technology (2015R1D1A1A09061368) and supported by the KIAT (Korea Institute for Advancement of Technology) grant funded by the Korea Government (MOTIE : Ministry of Trade Industry and Energy) (No. N0001884, HRD program for Embedded Software R\&D).

\section{References}

[1] S. H. Ahn, S. K. Kim, and K. H. Lee, "Development of a fleet management system for cooperation among construction equipment," Journal of the Korea Society of Civil Engineers, vol. 36, no. 3, pp. 573-586, 2016. https://doi.org/10.12652/Ksce.2016.36.3.0573

[2] F. David, P. Petr, M. Miroslav, and M. Milan, "Scanning of trucks to produce 3D models for analysis of timber loads," in Proceedings of 17th International Carpathian Control Conference (ICCC), Tatranska Lomnica, Slovakia, 2016, pp. 194-199. https://doi.org/10.1109/CarpathianCC.2016. 7501092

[3] D. Vrublova, R. Kapica, and J. Jurman, "Methodology devising for bucket-wheel excavator surveying by laser scanning method to determine its main geometrical parameters," Geodesy and Cartography, vol. 38, no. 4, pp. 157-164, 2012. https://doi.org/10.3846/20296991.2012. 757438

[4] J. U. Won, Y. S. Chung, W. S. Kim, K. H. You, Y. J. Lee, and K. H. Park, "A single camera based method for cubing rectangular parallelepiped objects," Journal of KIISE: Computing Practices and Letters, vol. 8, no. 5, pp. 562-573, 2002.
[5] Y. H. Baek and U. R. Moon, "Color edge detection using variable template operator," International Journal of Fuzzy Logic and Intelligent System, vol. 6, no. 2, pp. 116-120, 2006. https://doi.org/10.5391/IJFIS.2006.6.2.116

[6] Xing Xiong and Byung-Jae Choi, "Comparative analysis of detecting algorithms for corner and blob features in image processing," International Journal of Fuzzy Logic and Intelligent System, vol. 13, no. 4, pp. 284-290, Dec. 2013.

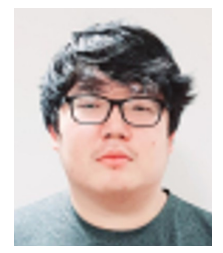

In-Hwan Kim has been under M.S. candidate course at Dongguk university, Korea, since 2016. His current research interests include robotics and intelligent human-robot interaction.

E-mail : dlsghks199@ naver.com

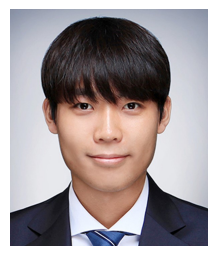

Dong-Woo Lim has been under M.S. candidate course at Dongguk university, Korea, since 2018. His current research interests include intelligent human-robot interaction and image processing.

E-mail : aehddn@gmail.com

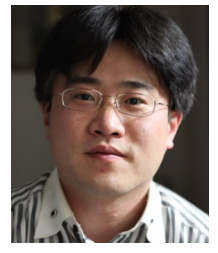

Jin-Woo Jung received the B.S. and M.S. degrees in electrical engineering from Korea Advanced Institute of Science and Technology (KAIST), Korea, in 1997 and 1999, respectively and received the $\mathrm{Ph} . \mathrm{D}$. degree in electrical engineering and computer science from KAIST, Korea in 2004. Since 2006, he has been with the Department of Computer Science and Engineering at Dongguk University, Korea, where he is currently a Professor. During 2001-2002, he worked as visiting researcher at the Department of MechanoInformatics, University of Tokyo, Japan. During 2004-2006, he worked as researcher in Human-friendly Welfare Robot System Research Center at KAIST, Korea. During 2014, he worked as visiting scholar at the Department of Computer and Information Technology, Purdue University, USA. His current research interests include human behavior recognition, multiple robot cooperation and intelligent human-robot interaction.

E-mail : jwjung@dongguk.edu 